\title{
$U$-EMBEDDED SUBSETS OF NORMED LINEAR SPACES
}

\author{
RONNIE LEVY AND M. D. RICE
}

\begin{abstract}
A subset $S$ of a metric space $X$ is $U$-embedded in $X$ if every uniformly continuous function $f: S \rightarrow R$ extends to a uniformly continuous function $F: X \rightarrow R$. Thus $U$-embedding is the uniform analogue of $C$-embedding. The Tietze extension theorem tells us exactly which subsets of metric spaces are $C$-embedded. The uniform analogue would tell us exactly which subsets of metric spaces are $U$-embedded. In this paper, a characterization of $U$-embedded subsets of the Euclidean plane (or any normed linear space) is given.
\end{abstract}

A subset $S$ of a uniform space $X$ is $U$-embedded in $X$ if every real-valued uniformly continuous function $f: S \rightarrow R$ extends to a uniformly continuous function $F: X \rightarrow R$. Thus, $U$-embedding is the uniform analogue of $C$-embedding in topological spaces. One consequence of the Tietze extension theorem is that a subset of a metric space is $C$-embedded if and only if it is closed. Unlike the topological situation, a characterization of $U$-embedded subsets of metric spaces seems quite complicated. In this paper, we characterize those subsets of normed linear spaces which are $U$-embedded. As is usual in such situations, it is the convexity which will help us.

1. Preliminary definitions and results. Suppose that $(X, d)$ is a metric space. If $a, b \in X$, and $\varepsilon>0$, then we say that $a$ and $b$ are $\varepsilon$-linked by $n$ links in $X$ if there exists $a=x_{0}, x_{1}, \ldots, x_{n}=b \in X$ such that $d\left(x_{k-1}, x_{k}\right) \leq \varepsilon$ for $k=1,2, \ldots, n$. The finite sequence $a=x_{0}, \ldots, x_{n}=b$ is called an $\varepsilon$-chain from $a$ to $b$. If there exists an $n$ such that $a$ and $b$ are $\varepsilon$-linked by $n$ links in $X$, we say that $a$ and $b$ are $\varepsilon$-linked in $X$. A metric space is uniformly connected if it is not the union of two nonempty subsets which are a positive distance apart. Clearly, every connected metric space is uniformly connected. It is not difficult to see that $X$ is uniformly connected if and only if for each $\varepsilon>0$ and for each $a$ and $b$ in $X, a$ and $b$ are $\varepsilon$-linked in $X$.

Suppose $(X, d)$ is a metric space and $S$ is a subset such that every two elements of $S$ are $\varepsilon$-linked in $S$, where $\varepsilon$ is a positive number. Define

$$
d_{\varepsilon}^{S}(a, b)=\inf \left\{\sum_{i=1}^{m} d\left(z_{i-1}, z_{i}\right): a=z_{0}, \ldots, z_{m}=b \text { is an } \varepsilon \text {-chain in } S\right\}
$$

and let

$$
m_{\varepsilon}^{S}(a, b)=\min \{m \text { : there exists an } \varepsilon \text {-chain in } S \text { from } a \text { to } b \text { having } m \text { links }\} \text {. }
$$

Then $d_{\varepsilon}^{S}(a, b)$ measures the shortest distance one has to travel between $a$ and $b$ given that each step taken is in $S$ and each step is at most $\varepsilon$ units long. On the

Received by the editors March 15, 1985 and, in revised form, July 22, 1985.

1980 Mathematics Subject Classification. Primary 54C20.

Key words and phrases. $U$-embedding, Lipschitz for large distances. 
other hand, $m_{\varepsilon}^{S}(a, b)$ gives the fewest number of steps of length at most $\varepsilon$ one must take to get from $a$ to $b$ provided that each step is in $S$. Fairly simple examples show that it is possible to have the sum of the distances along every $\varepsilon$-chain from $a$ to $b$ having $m_{\varepsilon}^{S}(a, b)$ links be bounded away from $d_{\varepsilon}^{S}(a, b)$. Now let

$$
\hat{r}_{\varepsilon}^{S}=\sup \left\{d_{\varepsilon}^{S}(a, b) / d(a, b): a, b \in S, a \neq b\right\}
$$

and let

$$
r_{\varepsilon}^{S}=\sup \left\{m_{\varepsilon}^{S}(a, b) / m_{\varepsilon}^{X}(a, b): a, b \in S, a \neq b\right\} .
$$

When the subspace $S$ is clear from the context, we omit the superscript $S$ in $\hat{r}_{\varepsilon}^{S}$ and $r_{\varepsilon}^{S}$.

1.1. Proposition. Suppose $(X, d)$ is a metric space and $S$ is a subspace of $X$. Then $r_{\varepsilon} \leq 4 \hat{r}_{\varepsilon}+2$. Therefore, if $\hat{r}_{\varepsilon}$ is finite, then $r_{\varepsilon}$ is finite.

Proof. Suppose $a$ and $b$ are in $S$. Given $\delta>0$, choose an $\varepsilon$-chain $a=$ $z_{0}, z_{1}, \ldots, z_{N}=b$ from $a$ to $b$ such that

$$
A=\sum_{i=1}^{N} d\left(z_{i-1}, z_{i}\right) \leq d_{\varepsilon}^{S}(a, b)+\delta
$$

where $N$ is the smallest integer for which there exists such an $\varepsilon$-chain. Then

$$
d\left(z_{2 k-2}, z_{2 k}\right)>\varepsilon \text { for } k=1, \ldots,[N / 2],
$$

because if this inequality did not hold, we could get an $\varepsilon$-chain having fewer links and the corresponding $\varepsilon$-chain would give a sum of distances not exceeding $A$. From (*), we get $A \geq[N / 2] \varepsilon / 2$, so

$$
[N / 2] \varepsilon / 2 \leq d_{\varepsilon}^{S}(a, b)+\delta .
$$

Set $m=m_{\varepsilon}^{S}(a, b)$. By the choice of $N$ and the definition of $m_{\varepsilon}^{S}(a, b), m \leq N$. Repeated applications of the triangle inequality give $d(a, b) \leq \varepsilon m_{\varepsilon}^{X}(a, b)$. Then we get

so

$$
\begin{aligned}
& m_{\varepsilon}^{S}(a, b) / m_{\varepsilon}^{X}(a, b)=\varepsilon m_{\varepsilon}^{S}(a, b) / \varepsilon m_{\varepsilon}^{X}(a, b) \\
& \quad \leq \varepsilon m_{\varepsilon}^{S}(a, b) / d(a, b)=\varepsilon m / d(a, b) \leq N \varepsilon / d(a, b),
\end{aligned}
$$

$$
\begin{aligned}
m_{\varepsilon}^{S}(a, b) / m_{\varepsilon}^{X}(a, b) & \leq N \varepsilon / d(a, b) \leq\left[4\left(d_{\varepsilon}^{S}(a, b)+\delta\right)+2 \varepsilon\right] / d(a, b) \\
& =4 d_{\varepsilon}^{S}(a, b) / d(a, b)+(4 \delta+2 \varepsilon) / d(a, b) .
\end{aligned}
$$

If $d(a, b) \geq \varepsilon$, then $m_{\varepsilon}^{S}(a, b) / m_{\varepsilon}^{X}(a, b) \leq 4 \hat{r}_{\varepsilon}+(4 \delta+2 \varepsilon) / \varepsilon=4 \hat{r}_{\varepsilon}+2+(4 \delta / \varepsilon)$. On the other hand, if $d(a, b)<\varepsilon$, then $m_{\varepsilon}^{S}(a, b)=m_{\varepsilon}^{X}(a, b)=1$ and $d_{\varepsilon}^{S}(a, b)=d(a, b)$, so $m_{\varepsilon}^{S}(a, b) / m_{\varepsilon}^{X}(a, b)=1=d_{\varepsilon}^{S}(a, b) / d(a, b)$. Therefore, $r_{\varepsilon} \leq 4 \hat{r}_{\varepsilon}+2+4 \delta / \varepsilon$ for each $\delta>0$, so $r_{\varepsilon} \leq 4 \hat{r}_{\varepsilon}+2$.

If $(X, d)$ is a metric space and $f: X \rightarrow R$ is a function, then $f$ is Lipschitz for large distances if for each $\varepsilon>0$, there exists a constant $K$ (which will in general depend upon $\varepsilon$ ) such that $d(x, y) \geq \varepsilon$ implies that $|f(x)-f(y)| \leq K d(x, y)$. If $F$ is a family of functions from $X$ to $R$, then $F$ is said to be jointly Lipschitz for large distances if for each $\varepsilon>0$ there exists a constant $K$ (depending upon $\varepsilon$ ) such that if $d(x, y) \geq \varepsilon$ and $f \in F$, then $|f(x)-f(y)| \leq K d(x, y)$. The phrase " $(X, d)$ is a normed linear space" will be used to mean that $d$ is the metric induced by the norm on the normed linear space $X$. 
1.2. LEMMA [LR $\mathbf{L R}_{1}$. If $(X, d)$ is a normed linear space and $S$ is a subset of $X$, then $S$ is $U$-embedded in $X$ if and only if each uniformly continuous function $f: S \rightarrow R$ is Lipschitz for large distances.

1.3. LEMMA $\left[\mathbf{L R}_{2}\right]$. If $(X, d)$ is a normed linear space and $S$ is a uniformly connected subset of $X$, then $S$ is $U$-embedded in $X$ if and only if each equi-uniformlycontinuous family $F$ of functions from $S$ to $R$ is jointly Lipschitz for large distances.

2. The uniformly connected case. In this section, we give a characterization of those uniformly connected subsets of normed linear spaces which are $U$-embedded. In the next section we show how to modify the characterization for the case where the subset $S$ is not assumed to be uniformly connected.

2.1. PROPOSITION. Suppose $(X, d)$ is a normed linear space, and suppose that $S$ is a uniformly connected $U$-embedded subset of $X$. Then $\hat{r}_{\varepsilon}$ is finite for each $\varepsilon>0$.

PROOF. Assume $\hat{r}_{\varepsilon}=+\infty$ for some $\varepsilon>0$. Then there exist sequences $\left(x_{k}\right)$ and $\left(y_{k}\right)$ of points of $S$ such that $x_{k} \neq y_{k}$ and $d_{\varepsilon}^{S}\left(x_{k}, y_{k}\right) \geq k d\left(x_{k}, y_{k}\right)$ for $k=1,2, \ldots$ Since $d(x, y) \leq \varepsilon$ implies that $d_{\varepsilon}^{S}(x, y)=d(x, y)$, the choice of the $x_{k}$ 's and $y_{k}$ 's gives us that $d\left(x_{k}, y_{k}\right)>\varepsilon$ for $k \geq 2$. For $k=1,2, \ldots$, define $g_{k}: S \rightarrow R$ by $g_{k}(x)=d_{\varepsilon}^{S}\left(x, y_{k}\right)$. We claim that the family $\left\{g_{k}: k=1,2, \ldots\right\}$ is equi-uniformly continuous. Choose $\eta>0$ and let $\delta=\min \{\eta, \varepsilon\}$. Suppose $x, y \in S$ and $d(x, y)<\delta$. Then $d(x, y) \leq \varepsilon$. Given $\rho>0$, choose $\varepsilon$-chains $y=y_{0}, y_{1}, \ldots, y_{L(k)}=y_{k}$ and $x=x_{0}, x_{1}, \ldots, x_{M(k)}=y_{k}$ such that

$$
\sum_{i=1}^{L(k)} d\left(y_{i-1}, y_{i}\right)<d_{\varepsilon}^{S}\left(y, y_{k}\right)+\rho
$$

and

$$
\sum_{j=1}^{M(k)} d\left(x_{j-1}, x_{j}\right)<d_{\varepsilon}^{S}\left(x, y_{k}\right)+\rho
$$

Then

$$
d_{\varepsilon}^{S}\left(x, y_{k}\right) \leq d(x, y)+\sum_{i=1}^{L(k)} d\left(y_{i-1}, y_{i}\right)<d(x, y)+d_{\varepsilon}^{S}\left(y, y_{k}\right)+\rho
$$

Therefore,

$$
d_{\varepsilon}^{S}\left(x, y_{k}\right)-d_{\varepsilon}^{S}\left(y, y_{k}\right)<d(x, y)<\eta
$$

Similarly, one shows that

$$
d_{\varepsilon}^{S}\left(y, y_{k}\right)-d_{\varepsilon}^{S}\left(x, y_{k}\right)<d(x, y)<\eta
$$

Therefore, $\left|g_{k}(x)-g_{k}(y)\right| \leq \eta$. This proves the claim. However, $\left|g_{k}\left(x_{k}\right)-g_{k}\left(y_{k}\right)\right|=$ $d_{\varepsilon}^{S}\left(x_{k}, y_{k}\right) \geq k d\left(x_{k}, y_{k}\right), k=1,2, \ldots$. Since $d\left(x_{k}, y_{k}\right)>\varepsilon$ for $k \geq 2$, this violates 1.3 .

2.2. Proposition. Suppose that $S$ is a uniformly connected subset of a normed linear space $(X, d)$. If $r_{\varepsilon}$ is finite for each positive $\varepsilon$, then $S$ is $U$-embedded in $X$.

ProOF. Suppose $f: S \rightarrow R$ is uniformly continuous. Suppose $\varepsilon>0$. We must find a constant $K_{\varepsilon}$ such that $d(x, y) \geq \varepsilon$ implies that $|f(x)-f(y)|<K_{\varepsilon} d(x, y)$. 
Choose $\delta>0$ such that $d(x, y)<\delta$ implies that $|f(x)-f(y)|<1$. Assume $d(x, y) \geq \varepsilon$. Let $m=m_{\delta}^{S}(x, y)$ and let $x=x_{0}, x_{1}, \ldots, x_{m}=y$ be a $\delta$-chain in $S$. Then if we let $K_{\varepsilon}=r_{\delta}[(\varepsilon+\delta) / \varepsilon \delta]$, we get

$$
\begin{aligned}
|f(x)-f(y)| & \leq \sum_{i=1}^{m}\left|f\left(x_{i-1}\right)-f\left(x_{i}\right)\right| \\
& \leq m=m_{\delta}^{X}(x, y)\left(m / m_{\delta}^{X}(x, y)\right) \\
& \left.\leq r_{\delta}[(d(x, y) / \delta)+1] \quad \text { (because } m_{\delta}^{X}(x, y)=[(d(x, y) / \delta)+1]\right) \\
& \leq r_{\delta}\{d(x, y)+(\delta / \varepsilon) \varepsilon\} / \delta \\
& \left.\leq r_{\delta}\{(\varepsilon d(x, y)+\delta d(x, y)) / \varepsilon \delta\} \quad \text { (since } \varepsilon \leq d(x, y)\right) \\
& =K_{\varepsilon} d(x, y) .
\end{aligned}
$$

Therefore, $f$ is Lipschitz for large distances.

Combining 1.1 with the preceding two propositions gives the following theorem:

2.3. THEOREM. Suppose that $S$ is a uniformly connected subset of the normed linear space $X$. Then the following are equivalent:

(i) $\hat{r}_{\varepsilon}$ is finite for each $\varepsilon>0$.

(ii) $r_{\varepsilon}$ is finite for each $\varepsilon>0$.

(iii) $S$ is $U$-embedded in $X$.

3. The general case. In this section, we give a characterization of those subsets of normed linear spaces which are $U$-embedded. The characterization will use the characterization given in $\S 2$ for the case where the subspace is uniformly connected.

3.1. Proposition. Suppose $S$ is a $U$-embedded subset of the normed linear space $(X, d)$. Then for each $\varepsilon>0$ there exists a compact subset $F$ of $X$, which may be taken to be the union of finitely many line segments, such that

(i) every two elements of $S \cup F$ are $\varepsilon$-linked in $S \cup F$,

(ii) $\hat{r}_{\varepsilon}^{S \cup F}$, and therefore $r_{\varepsilon}^{S \cup F}$, is finite.

Proof. For each $p \in S$, define $C_{p}=\{x \in S: x$ and $p$ are $\varepsilon / 3$-linked in $S\}$. If $C_{p} \neq C_{q}$, then $d\left(C_{p}, C_{q}\right) \geq \varepsilon / 3$, so $C=\left\{C_{p}: p \in S\right\}$ is a uniformly discrete family of nonempty subsets of $X$ whose union is the $U$-embedded set $S$. Therefore, $C$ is finite. (See $\left[\mathbf{L R}_{1}\right]$.) Write $C=\left\{D_{0}, D_{1}, \ldots, D_{N}\right\}$ and for each $k=0,1, \ldots, N$ choose $p_{k} \in D_{k}$. For $k=1,2, \ldots, N$, let $F_{k}$ be the line segment from $p_{k-1}$ to $p_{k}$ and let $F=\bigcup_{k=1}^{N} F_{k}$. With this definition, condition (i) is clearly satisfied. Now let $\hat{S}=\{p \in X: d(p, S \cup F) \leq \varepsilon / 3\}$. It is easy to see that $\hat{S}$ is uniformly connected. We claim that every uniformly continuous function $g: \hat{S} \rightarrow R$ which is identically zero on $S$ is bounded. It will then follow from $\left[\mathbf{L R}_{1}\right]$ that $\hat{S}$ is $U$-embedded in $X$. So assume $g: \hat{S} \rightarrow R$ is uniformly continuous and identically zero on $S$. Since $F$ is compact, it follows that the restriction of $g$ to $S \cup F$ is bounded. Let $K$ be an upper bound for the absolute value of this restriction. Now let $\delta>0$ be such that $x, y \in S \cup F, d(x, y)<\delta$ imply $|g(x)-g(y)| \leq 1$. Given a point $x$ of $\hat{S}$, there exists a point $p$ of $S \cup F$ such that the segment from $x$ to $p$ is contained in $\hat{S}$ and has length at most $\varepsilon / 3$. Then $|g(x)| \leq B+K$, where $B=[(\varepsilon / 3 \delta)+1]$. Therefore, $\hat{S}$ is $U$-embedded in $X$. 
It follows from 2.3 that $\hat{r}_{\varepsilon / 3}^{S}$ is finite. We will show that this implies that $\hat{r}_{\varepsilon}^{S \cup F}$ is finite. Suppose $a, b \in S \cup F$. Given $\rho>0$, choose an $\varepsilon / 3$-chain $a=a_{0}, a_{1}, \ldots, a_{M}=$ $b$ in $\hat{S}$ such that

$$
A=\sum_{i=1}^{M} d\left(a_{i-1}, a_{k}\right)<d_{\varepsilon / 3}^{S}(a, b)+\rho .
$$

We may assume without loss of generality that this $\varepsilon / 3$ chain is minimal in the sense that $d\left(a_{0}, a_{2}\right)>\varepsilon / 3, d\left(a_{2}, a_{4}\right)>\varepsilon / 3, \ldots$. (If this is not the case, inductively choose the elements of the chain so that the resulting chain is minimal and (\#) will still hold.) Then at least half of the distances $d\left(a_{i-1}, a_{i}\right)$ are at least $\varepsilon / 6$, so

$$
A \geq(\varepsilon / 6)[M / 2], \quad \text { that is, } \quad M \leq(12 A / \varepsilon)+2 .
$$

For each $k=1,2, \ldots, M-1$, choose $x_{k} \in S \cup F$ such that $d\left(x_{k}, a_{k}\right)<\varepsilon / 3$. Then if $x_{0}=a$ and $x_{M}=b$, one gets that $d\left(x_{k-1}, x_{k}\right) \leq d\left(x_{k-1}, a_{k-1}\right)+d\left(a_{k-1}, a_{k}\right)+$ $d\left(a_{k}, x_{k}\right) \leq d\left(a_{k-1}, a_{k}\right)+2 \varepsilon / 3 \leq \varepsilon / 3+2 \varepsilon / 3=\varepsilon$, so $a=x_{0}, x_{1}, \ldots, x_{M}=b$ is an $\varepsilon$-chain in $S \cup F$. Furthermore,

$$
\sum_{i=1}^{M} d\left(x_{i-1}, x_{i}\right) \leq \sum_{i=1}^{M} d\left(a_{i-1}, a_{i}\right)+2 M \varepsilon / 3 .
$$

Therefore, using $(*)$ we get $\sum_{i=1}^{M} d\left(x_{i-1}, x_{i}\right) \leq A+(2 \varepsilon / 3)[(12 A / \varepsilon)+2]=9 A+$ $(4 \varepsilon / 3) \leq 9 d_{\varepsilon / 3}^{S}(a, b)+[9 \rho+(4 \varepsilon / 3)]$. Therefore, $d_{\varepsilon}^{S \cup F}(a, b) \leq 9 d_{\varepsilon / 3}^{S}(a, b)+(4 \varepsilon / 3)$. Hence, $\hat{r}_{\varepsilon}^{S \cup F} \leq 9 \hat{r}_{\varepsilon / 3}^{S}+(4 \varepsilon / 3)$.

3.2. Proposition. Suppose $S$ is a subset of a normed linear space $X$. Suppose that for each $\varepsilon>0$ there exists a compact set $F$ such that

(i) every two elements of $S \cup F$ are $\varepsilon$-linked in $S \cup F$,

(ii) $r_{\varepsilon}^{S \cup F}$ is finite.

Then $S$ is $U$-embedded in $X$.

Proof. Suppose $f: S \rightarrow R$ is uniformly continuous. By a theorem of Isbell [I], there exists an $\varepsilon>0$ such that $f$ can be extended to a uniformly continuous function $f_{1}: S_{\varepsilon} \rightarrow R$, where $S_{\varepsilon}=\{x \in X: d(x, S) \leq \varepsilon\}$. Choose a positive $\delta<\varepsilon$ such that $x, y \in S_{\varepsilon}$ and $d(x, y)<\delta$ imply that $\left|f_{1}(x)-f_{1}(y)\right|<1$. Let $F$ be the compact set given by the hypothesis corresponding to $\delta$. By $\left[\mathbf{L R}_{1}\right], f_{1}$ can be extended to a uniformly continuous function $\hat{f}: S_{\varepsilon} \cup F \rightarrow R$. We claim that there exists a constant $C$ such that if $x$ and $y$ are elements of $S \cup F$ satisfying $d(x, y)<\delta$, then $|\hat{f}(x)-\hat{f}(y)| \leq C$. Let $M$ be a constant such that $|f(x)|<M$ for all $x$ in $F$. Suppose $x, y \in S \cup F$ and $d(x, y)<\delta$. If $\{x, y\} \subseteq F$, then $|f(x)-f(y)| \leq 2 M$. If $\{x, y\} \subseteq S$, then $|f(x)-f(y)|<1$. If $x \in S, y \in F$, then $y \in S_{\varepsilon}$ (because $d(x, y)<\varepsilon$ ) so again we have $|f(x)-f(y)|<1$. Therefore, we may choose $C=2 M+1$.

Now assume $d(x, y) \geq \varepsilon$, where $x$ and $y$ are points of $S \cup F$. Let $m=m_{\delta}^{S \cup F}(x, y)$ and let $x=x_{0}, x_{1}, \ldots, x_{m}=y$ be a $\delta$-chain in $S \cup F$. Then

$$
\begin{aligned}
|f(x)-f(y)| & \leq \sum_{i=1}^{m}\left|f\left(x_{i-1}\right)-f\left(x_{i}\right)\right| \leq m C \\
& \leq C r_{\delta}^{S \cup F}[(\varepsilon+\delta) / \varepsilon \delta] d(x, y)=K_{\varepsilon} d(x, y),
\end{aligned}
$$




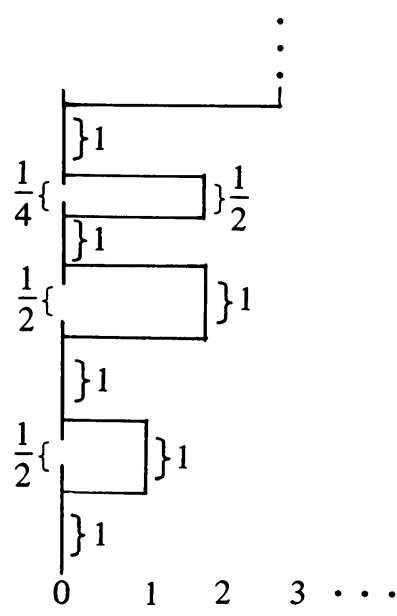

$X$

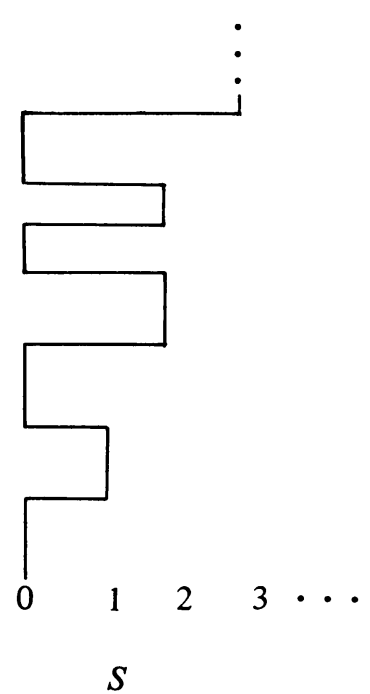

$S$

Diagram

where $K_{\varepsilon}=C r_{\delta}^{S \cup F}(\varepsilon+\delta) / \varepsilon \delta$. Therefore, $f$ is Lipschitz for large distances.

Combining 3.1 and 3.2 gives the following theorem:

3.3. THEOREM. Suppose $S$ is a subset of the normed linear space $(X, d)$. Then the following are equivalent:

(i) $S$ is $U$-embedded in $X$,

(ii) for each $\varepsilon>0$, there exists a compact set $F$ (which may be taken to be a union of finitely many line segments) such that any two elements of $S \cup F$ are $\varepsilon$-linked in $S \cup F$ and such that $r_{\varepsilon}^{S \cup F}$ is finite.

REMARKS. 1. If $(X, d)$ is any uniformly connected metric space, then by embedding $X$ isometrically in a normed linear space and appealing to 1.2 and 2.3 one sees that if every uniformly continuous function $f: X \rightarrow R$ is Lipschitz for large distances, then $r_{\varepsilon}^{X}$ is finite for each positive $\varepsilon$. In fact, the converse of this statement is true as well: If $r_{\varepsilon}^{X}$ is finite for each positive $\varepsilon$, then every uniformly continuous function $f: X \rightarrow R$ is Lipschitz for large distances. Since there are easy examples of metric spaces where not every uniformly continuous real-valued function is Lipschitz for large distances, this means that the finiteness of $r_{\varepsilon}^{S}$ for each positive $\varepsilon$ is not in general equivalent to the $U$-embedding of a subspace $S$ of a space $X$.

2. We do not know an example of a metric space $(X, d)$ and a non- $U$-embedded uniformly connected subset $S$ such that $r_{\varepsilon}^{S}$ is finite for each positive $\varepsilon$. However, the following example shows that a uniformly connected, $U$-embedded subset of a metric space can have infinite $r_{\varepsilon}^{S}$ for all sufficiently small $\varepsilon>0$. 
3.4. EXAMPLE. There exists a (uniformly) connected metric space $X \subset R^{2}$ and a $U$-embedded (uniformly) connected subset $S$ such that $r_{\varepsilon}=+\infty$ for all positive $\varepsilon<1 / 2$. Rather than give a description of $X$ and $S$, we will draw pictures. Any uniformly continuous $f: S \rightarrow R$ can be extended to a uniformly continuous function $F: X \rightarrow R$ by making $F$ constant on the small vertical whiskers growing along the $y$-axis. By restricting our attention to those horizontal bands of $S$ of height $1 / n$, one can show that $r_{1 /(2 n)}=\infty$ for $n=1,2, \ldots$. For an arbitrary positive $\varepsilon \leq 1 / 2$, find an $n$ satisfying $1 /(2 n)<\varepsilon<1 / n$ (that is, $1 / 2<n \varepsilon \leq 1$ ), and by restricting our attention to the horizontal bands of height $1 / n$, we can again show that $r_{\varepsilon}=\infty$.

REMARKS. 1. By modifying the construction in 3.4 , it is possible to find a connected metric space $X$ and a connected $U$-embedded subset $S$ such that $r_{\varepsilon}=\infty$ for all $\varepsilon>0$.

2. By appealing to the results in $\left[\mathbf{L R}_{3}\right]$ the results in this paper also give results about Hilbert spaces. For example, it is shown in $\left[\mathbf{L R}_{3}\right]$ that a subset $S$ of a Hilbert space $H$ is $U$-embedded in $H$ if and only if every uniformly continuous function $f: S \rightarrow H$ extends to a uniformly continuous function $F: H \rightarrow H$. Thus, we also have characterized those subsets of Hilbert space for which every uniformly continuous function into a Hilbert space extends to a uniformly continuous function.

\section{REFERENCES}

[I] John Isbell, Uniform spaces, Math. Surveys, no. 12, Amer. Math. Soc., Providence, R. I., 1964.

[LR $\left.\mathbf{L}_{1}\right]$ Ronnie Levy and M. D. Rice, Techniques and examples in U-embedding, Topology Appl. (to appear).

$\left[\mathbf{L R}_{2}\right] \ldots$, The extension of equi-uniformly continuous families of mappings, Pacific J. Math. 12 (1985), 149-161.

$\left[\mathbf{L R}_{3}\right]$, The approximation and extension of uniformly continuous Banach space valued mappings, Comment. Math. Univ. Carolin. 24 (1983), 251-265.

Department of Mathematical SCiences, George Mason University, Fairfax, VIRGINIA 22030

Department of Computer Science, George Mason University, Fairfax, VirGINIA 22030 